\title{
RF LOADS FOR THE CLIC MULTIBUNCH STRUCTURE
}

\author{
M. Luong, I. Wilson, W. Wuensch, CERN, 1211 Geneva 23, Switzerland
}

\begin{abstract}
The CLIC multibunch accelerating structure, called the TDS (Tapered Damped Structure), relies on heavy damping of higher-order modes [1]. Each cell of the structure is damped by its own set of four individuallyterminated waveguides. A compact, low-cost and largebandwidth terminating load with a reflection coefficient below 0.05 has been developed for this application. The load consists of a silicon carbide pyramid centered in a tapered waveguide. The design process, including the technique used to measure complex permittivity, is described. Measurements made on scaled prototypes are presented.
\end{abstract}

\section{INTRODUCTION}

Beam dynamics simulations of the CLIC main linac have shown that in order to preserve small emittances a substantial transverse wakefield suppression is required in accelerating structures. The required suppression is achieved in the TDS design by strong damping and moderate detuning.

Each cell of the TDS is damped by its own set of four waveguides which produce a quality factor of about 16 for the lowest transverse passband. The cutoff frequency of the waveguides $(33.5 \mathrm{GHz})$ is chosen to lie above the accelerating frequency $(29.985 \mathrm{GHz})$ but below the transverse mode spectrum (starting from about $36 \mathrm{GHz}$ ). The waveguide acts as a high-pass filter - higher order modes are damped while the accelerating mode is not.

Each damping waveguide is individually terminated by an absorbing load - a reflection coefficient less than 0.05 over a large bandwidth (extending to near the waveguide cutoff frequency) is required for effective damping. The terminating load must also satisfy a number of technical constraints - it must be compact, mechanically simple, brazeable, ultra-high vacuum compatible, radiation resistant and inexpensive. A load that fulfills these design criteria has been developed. The design process and the performance of a scaled prototype load are presented here.

\section{SILICON CARBIDE AND THE MEASUREMENT OF PERMITTIVITY}

The RF design of the termination depends strongly on the permittivity of the absorbing element. Following the example of other accelerator applications, $\mathrm{SiC}$ has been chosen as the absorbing material. $\mathrm{SiC}$ satisfies the technical constraints listed above and is widely-available, low-cost and used in diverse industrial applications. There is, however, little published data on the high frequency permittivity of $\mathrm{SiC}$ and no specific data available from individual suppliers. In order to validate the choice of $\mathrm{SiC}$ and then to design the load, a simple technique to measure permittivity has been developed.

The technique involves measuring the complex reflection coefficient from a small slab of the material under test. The permittivity is obtained by comparing the measured reflection coefficient to the reflection coefficient computed on the same geometry but with an estimated permittivity. An increasingly accurate estimate of complex permittivity is obtained through iterative optimization.

A typical setup used to produce experimental reflection coefficient data for $\mathrm{SiC}$ is shown in Fig. 1 and data was taken at other frequencies using similar setups. The shape of the slab is not important but its dimensions must be accurately measured for setup of the computer simulation. The experimental setup has no contacts between the $\mathrm{SiC}$ and the copper except where the back plane of the $\mathrm{SiC}$ is glued with epoxy to the waveguide short circuit. The contact does not influence the reflection coefficient since the fields are already attenuated by the $\mathrm{SiC}$. The complex reflection coefficient, $\Gamma_{\mathrm{m}}$, is measured in the usual way using an HP8510B network analyzer. Reflection data for two different thickness SiC slabs are shown in Figs. 2 and 3. The $S_{11}$-port calibration provides an accuracy better than 0.01 for the amplitude and 0.5 degrees for the phase of the reflection coefficient.

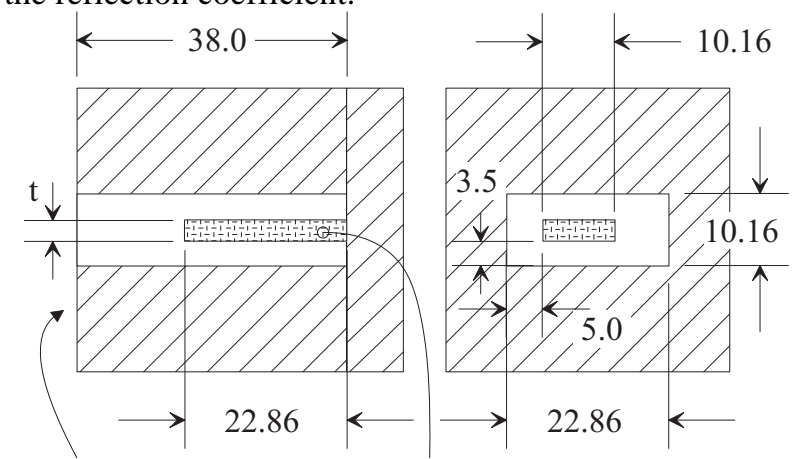

Reference plane Dissipative material (SiC)

Figure 1: X-band experimental setup (dimensions in $\mathrm{mm}$ ).

The notation for complex relative permittivity that will be used is the one based on a real relative dielectric constant, $\varepsilon^{\prime}$, and a loss tangent, $\tan \delta$.

$$
\varepsilon=\varepsilon^{\prime}+i \varepsilon^{\prime \prime}=\varepsilon^{\prime}(1+i \tan \delta)
$$

It is assumed that $\mathrm{SiC}$ has relative permeability, $\mu=1$. 


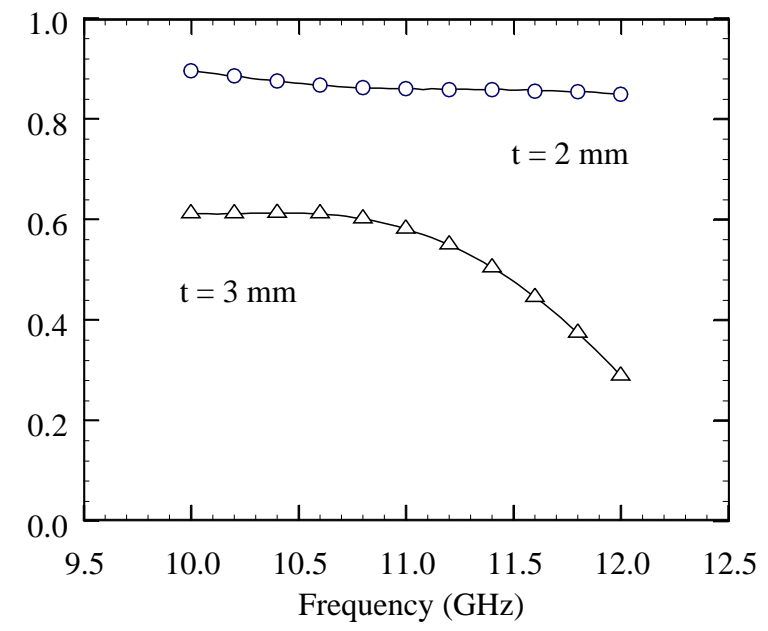

Figure 2: Amplitudes of measured reflection coefficients.

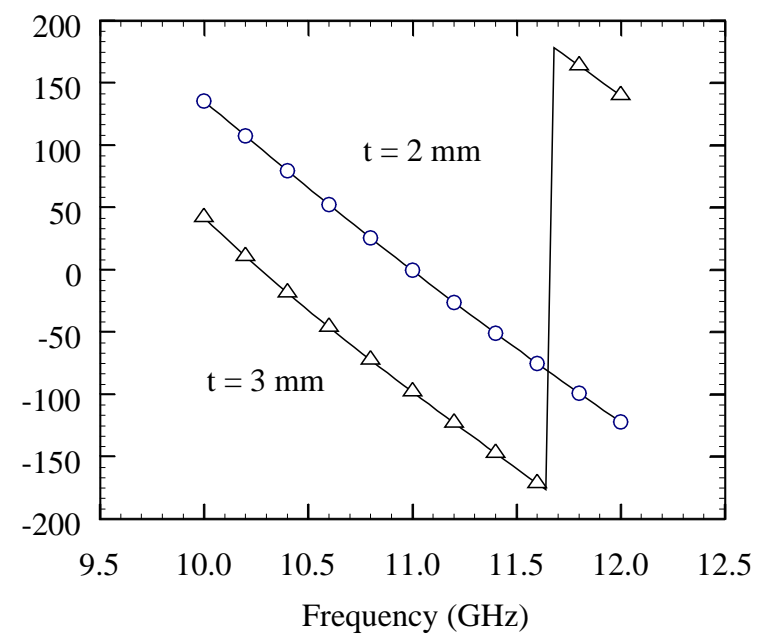

Figure 3: Phases of measured reflection coefficients.

The reflection coefficients were computed using the program HFSS [2]. An initial reflection coefficient is computed for each frequency using a starting value of $\varepsilon$. A new $\varepsilon$ estimate at each frequency is found by minimizing the error between measured and computed reflection coefficients using the Newton algorithm. This algorithm converges quickly as long as the seed values are not too far from the solution. The two equations to be minimized are:

$a\left(\varepsilon^{\prime}, \tan \delta\right)=\operatorname{real}\left\{\Gamma_{c}\left(\varepsilon^{\prime}, \tan \delta\right)-\Gamma_{m}\right\}=0$,

$b\left(\varepsilon^{\prime}, \tan \delta\right)=\operatorname{imag}\left\{\Gamma_{c}\left(\varepsilon^{\prime}, \tan \delta\right)-\Gamma_{m}\right\}=0$.

The Newton algorithm gives incremental steps, $\mathrm{d} \varepsilon^{\prime}$ and dtan $\delta$, between iterations of,

$$
\begin{aligned}
& {\left[\begin{array}{l}
\frac{\partial a\left(\varepsilon_{n}^{\prime}, \tan \delta_{n}\right)}{\partial \varepsilon^{\prime}} \frac{\partial a\left(\varepsilon_{n}^{\prime}, \tan \delta_{n}\right)}{\partial \tan \delta} \\
\frac{\partial b\left(\varepsilon_{n}^{\prime}, \tan \delta_{n}\right)}{\partial \varepsilon^{\prime}} \frac{\partial b\left(\varepsilon_{n}^{\prime}, \tan \delta_{n}\right)}{\partial \tan \delta}
\end{array}\right]\left[\begin{array}{l}
d \varepsilon^{\prime} \\
d \tan \delta
\end{array}\right]=} \\
& -\left[\begin{array}{l}
a\left(\varepsilon_{n}^{\prime}, \tan \delta_{n}\right) \\
b\left(\varepsilon_{n}^{\prime}, \tan \delta_{n}\right)
\end{array}\right] .
\end{aligned}
$$

Partial derivatives are obtained by numerical approximation :

$$
\frac{\partial \mathrm{F}\left(\mathrm{x}_{\mathrm{i}}, \ldots\right)}{\partial \mathrm{x}_{\mathrm{i}}} \cong \frac{\mathrm{F}\left(\mathrm{x}_{\mathrm{i}}+\mathrm{h}, \ldots\right)-\mathrm{F}\left(\mathrm{x}_{\mathrm{i}}, \ldots\right)}{\mathrm{h}},
$$

where $\mathrm{h}=0.05 \mathrm{x}_{\mathrm{i}}$ is a practical value. The algorithm is implemented in a $\mathrm{C}$ routine in which HFSS is called as a subroutine with input parameters $\varepsilon^{\prime}$ and $\tan \delta$. The algorithm is stopped once $a\left(\varepsilon^{\prime}, \Delta\right)$ and $b\left(\varepsilon^{\prime}, \Delta\right)$ are less than 0.01 . With seed values of 20 and 0.2 for $\varepsilon^{\prime}$ and $\tan \delta$ respectively, the algorithm converges within four passes.

An accurate value of permittivity requires a judicious compromise in the size of the SiC sample. The sample must be large enough to absorb a significant amount of energy but must also be small enough that the reflection is not dominated by the reflection from the front of the sample. Nonetheless the technique is insensitive to sample geometry. Raw and analyzed X-band measurements for samples 2 and $3 \mathrm{~mm}$ thick are shown in Figs. 2 to 5. Despite the large changes in raw reflection coefficients, the estimation technique converges on values of permittivity that agree to within $5 \%$.

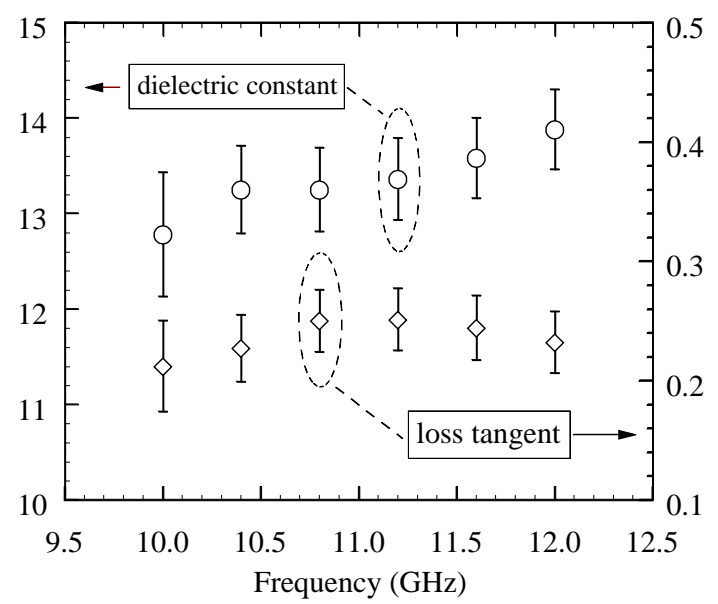

Figure 4: Permittivity values extracted from data from a $2 \mathrm{~mm}$ thick $\mathrm{SiC}$ sample.

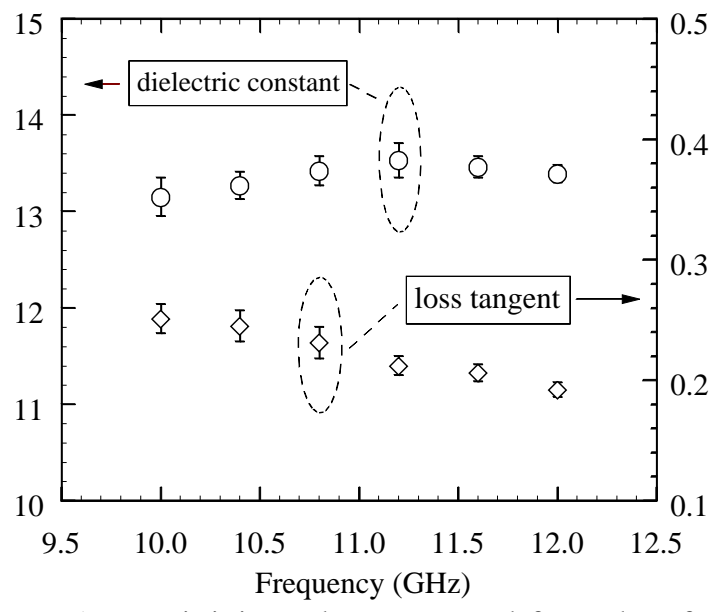

Figure 5: Permittivity values extracted from data from a $3 \mathrm{~mm}$ thick $\mathrm{SiC}$ sample. 


\section{PERMITTIVITY OF SiC}

SiC-100, manufactured by Ceramiques et Composites, has been chosen for use in TDS prototype studies. SiC100 has a high density $>3.1 \mathrm{~g} / \mathrm{cm}^{3}$. A compilation of complex permittivity data taken over a number of frequencies is shown in Figure 6.

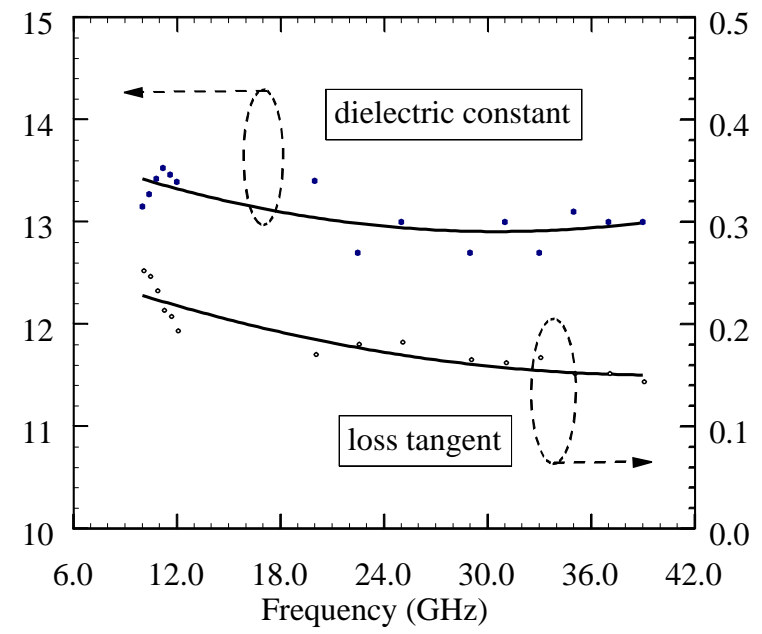

Figure 6: Complex permittivity of SiC-100.

\section{DESIGN OF THE LOAD}

The main requirements for the load: a reflection coefficient better than 0.05 for frequencies beyond 1.05 times the cutoff frequency of the damping waveguide and as good a performance as possible close to the cutoff. The load should be as short as possible and must have a simple form because many millions must be manufactured for CLIC. With the permittivity determined it was possible to use the program HFSS to compare various design principles and also to determine final dimensions.

The performance away from cutoff is easily achieved using a pyramidal tapered load. Near cutoff however, the relative impedance mismatch caused by the $\mathrm{SiC}$ rises quickly. Load designs based on three ideas were pursued to decrease the mismatch near cutoff. In the first, the width of the guide is tapered out before introducing the load material - thus moving the cutoff mismatch of the $\mathrm{SiC}$ away from the passband of the waveguide. Such a load has a low reflection coefficient but is quite long. In the second, a pair of pyramids are placed along the sides of the waveguide in the zone where the waveguide is tapering out. Impedance variations are reduced by smoothing the effective width changes of the guide. The load has a good reflection coefficient and is short but each load requires two pieces of $\mathrm{SiC}$. In the third design, the waveguide is tapered in at the same time the $\mathrm{SiC}$ tapers out. The impedance change caused by the $\mathrm{SiC}$ is compensated by the impedance change of the narrowing guide. The third solution gives the best compromise of simplicity, size and performance and was thus chosen.
The load for the $30 \mathrm{GHz}$ TDS has not yet been designed because of the limited frequency range of the available network analyzer. A factor 3.5 scaled prototype has been made in preparation for a planned test of a scaled TDS structure in ASSET. The measured performance of the load is shown in figure 8. It should be noted that some care must be used to extract $S_{11}$ near the cutoff of the waveguide - a calibration with a reference plane inside the damping waveguide can be made using a pair of tapers and straight section of damping waveguide.

The agreement between expected and measured performances is excellent. The only differences are where the return loss is above $30 \mathrm{~dB}$. In addition this load achieves all the required RF performance objectives.

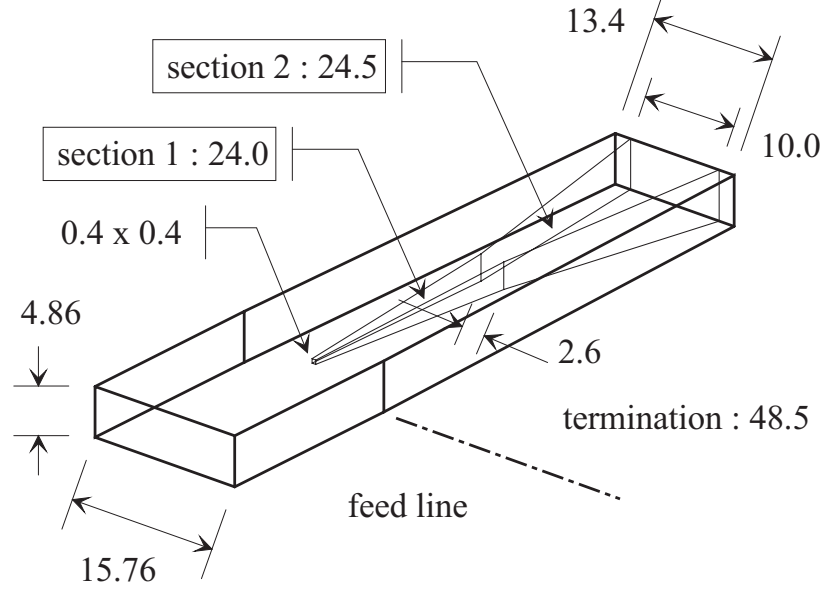

Figure 7: Load design .

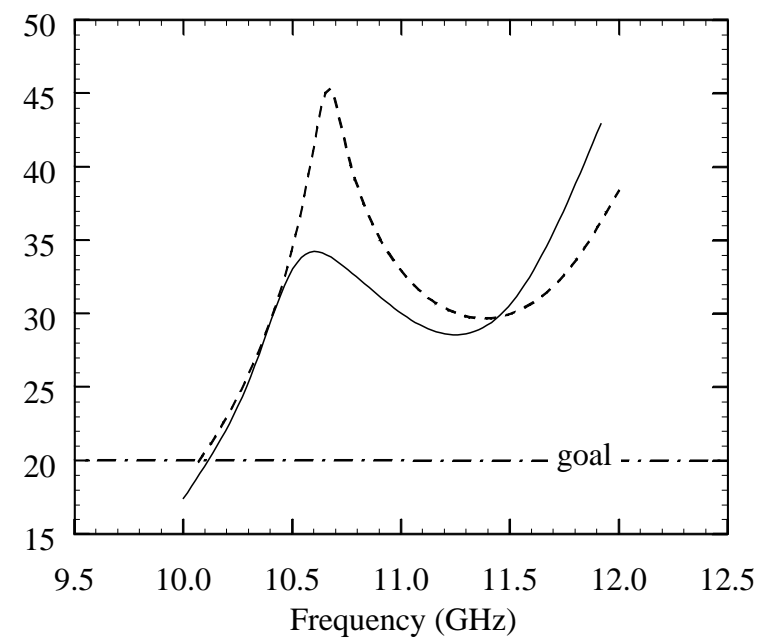

Figure 8: Measured (solid) and computed (dashed) load return loss as a function of frequency (in $\mathrm{dB}$ ).

\section{REFERENCES}

[1] M. Dehler, I. Wilson, W. Wuensch, 'A Tapered Damped Accelerating Structure for CLIC', LINAC98, Chicago, August 1998.

[2] HFSS, 'High Frequency Structure Simulator', Ansoft and Hewlett Packard Co.'s. 